\title{
Dscam1 Is Required for Normal Dendrite Growth and Branching But Not for Dendritic Spacing in Drosophila Motoneurons
}

\author{
Katie M. Hutchinson, ${ }^{1}$ Fernando Vonhoff, ${ }^{1,2}$ and Carsten Duch ${ }^{1,3}$ \\ ${ }^{1}$ School of Life Sciences, Arizona State University, Tempe, Arizona 85287, ${ }^{2}$ Department of Molecular, Cellular and Developmental Biology, Yale University, \\ New Haven, Connecticut 06520, and 3'Institute of Neurobiology, Johannes Gutenberg University of Mainz, 55099 Mainz, Germany
}

\begin{abstract}
Down syndrome cell adhesion molecule, Dscam, serves diverse neurodevelopmental functions, including axon guidance and synaptic adhesion, as well as self-recognition and self-avoidance, depending on the neuron type, brain region, or species under investigation. In Drosophila, the extensive molecular diversity that results from alternative splicing of Dscam 1 into $>38,000$ isoforms provides neurons with a unique molecular code for self-recognition in the nervous system. Each neuron produces only a small subset of Dscam1 isoforms, and distinct Dscam1 isoforms mediate homophilic interactions, which in turn, result in repulsion and even spacing of self-processes, while allowing contact with neighboring cells. While these mechanisms have been shown to underlie mushroom body development and spacing of mechanosensory neuron dendrites, here we report that Dscam1 plays no role in adult Drosophila motoneuron dendrite spacing, but is required for motoneuron dendritic growth. Targeted expression of Dscam-RNAi in an identified flight motoneuron did not impact dendrite spacing, but instead produced overgrowth. Increasing the knockdown strength severely reduced dendritic growth and branching. Similarly, Dscam mutant motoneurons in an otherwise control background (MARCM) were completely devoid of mature dendrites. These data suggest that Dscam1 is required cell autonomously for normal adult motoneuron dendrite growth in Drosophila. This demonstrates a previously unreported role of Drosophila Dscam1 in central neuron development, and expands the current understanding that Dscam1 operates as a cell adhesion molecule that mediates homophilic repulsion.
\end{abstract}

Key words: dendrite; development; Drosophila; Dscam; metamorphosis; motoneuron

\section{Introduction}

Dendritic architecture provides the structural blueprint for functional neural circuitry (Koch and Segev, 2000; Cline, 2001; Libersat and Duch, 2004; London and Häusser, 2005). During development dendritic architecture is regulated by innate genetic factors (Montague and Friedlander, 1989; Scott et al., 2003; Spatkowski and Schilling, 2003), growth factors and hormones (Weeks and Levine, 1995; Toran-Allerand et al., 1999; Cooke and Woolley, 2005), neuronal activity (Wong and Ghosh, 2002; Duch and Mentel, 2004; Vonhoff et al., 2013), guidance cues (Polleux et al., 2000; Kim and Chiba, 2004), and neuronal cell adhesion molecules (NCAMs; Williams et al., 2010).

Correct dendrite development requires precise control over rates of growth and branching, territory borders, and the com-

\footnotetext{
Received Aug. 8, 2013; revised Nov. 29, 2013; accepted Dec. 30, 2013.

Author contributions: C.D. designed research; K.M.H., F.V., and C.D. performed research; K.M.H. and F.V. analyzed data; K.M.H. and C.D. wrote the paper.

Support from the National Institutes of Health (1R01NS072128-01A1 to CD) is gratefully acknowledged. We thank Dr. Wesley Gruber, Columbia University, New York, for generously providing us with MARCM-ready fly stocks to conduct analysis of Dscam mutant motoneurons. We thank Dr. S. Ryglewski (Mainz, Germany) for help with numerous experiments, many helpful discussions, and comments on this manuscript.

The authors declare no competing financial interests.

Corresponding author: Katie Hutchinson, School of Life Sciences, Arizona State University, Tempe, AZ 85287. E-mail:kmhutchinson9@gmail.com.

DOI:10.1523/JNEUROSCI.3448-13.2014

Copyright $\odot 2014$ the authors $\quad 0270-6474 / 14 / 341924-08 \$ 15.00 / 0$
}

plete but nonredundant coverage of the territory. The latter requires that branches of each dendritic arbor be evenly spaced. Even spacing can be produced by repulsion among sister branches within a neuron, a phenomenon known as dendritic self-avoidance (Kramer and Kuwada, 1983; Kramer and Stent, 1985). However, in densely packed neuropils evenly spaced dendrites of one neuron must intermingle with the dendrites of other neurons. As such, mechanisms must exist to distinguish self from non-self dendrites.

In Drosophila, significant insights into self-recognition and self-avoidance have come from studies of the Ig superfamily molecule Down syndrome cell adhesion molecule (Dscam1). Alternative splicing of Dscam1 produces $>38,000$ isoforms (Schmucker et al., 2000). Identical splice variants interact strongly and mediate homophilic repulsion, but interactions between different isoforms are weak (Wojtowicz et al., 2007). Each neuron produces only $14-50$ isoforms so that differential splicing provides a self-recognition code (Hattori et al., 2007). In Drosophila numerous elegant studies demonstrate that Dscam1mediated homophilic repulsion is essential for the even spacing of dendrites of mechanosensory dendritic arborization (da) neurons (Grueber et al., 2002; Sweeney et al., 2002; Hughes et al., 2007; Matthews et al., 2007; Soba et al., 2007), and for correct dendritic patterning of olfactory projection neurons (OPN; Zhu et al., 2006). Likewise, vertebrate DSCAM governs dendrite self- 
A

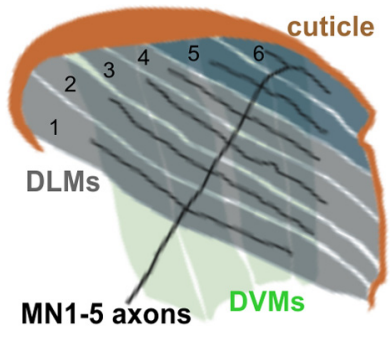

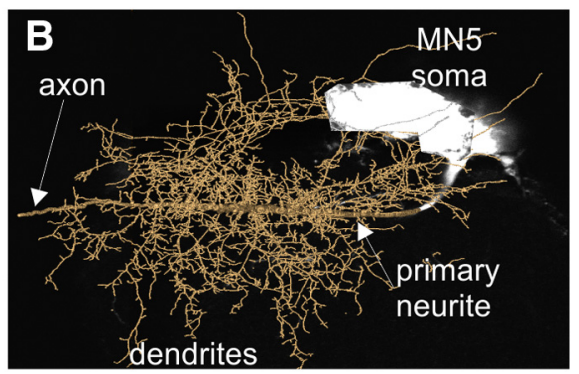

Figure 1. Motoneuron 5 and its target muscle fibers. A, Schematic drawing of the Drosophila flight muscle fibers, dorsoventral (DVM) and dorsolongitudinal (DLM). The DLM consists of six muscle fibers that are selectively innervated by motoneurons 1-5. $\boldsymbol{B}$, The fine structure of motoneuron 5, MN5, which innervates DLM muscle fibers 5 and 6.

avoidance and mosaic tiling in the retina of mice (Fuerst et al., 2008, 2009).

However, additional Dscam functions have been reported for directing lamina-specific synaptic connections in chick retina (Yamagata and Sanes, 2008), for interacting with netrins in axonal targeting (Andrews et al., 2008; Ly et al., 2008; Liu et al., 2009), for synaptic targeting via interactions with the FMRP protein (Cvetkovska et al., 2013; Kim et al., 2013), and for dendrite and spine morphogenesis in cortical pyramidal neurons (Maynard and Stein, 2012).

Here, we have used the well described and stereotyped dendritic architecture of the identified motoneuron, MN5 (Vonhoff and Duch, 2010; Kuehn and Duch, 2012; Vonhoff et al., 2012, 2013), to probe for the role of Dscam 1 in Drosophila central neuron dendrite development. We provide evidence that Dscam 1 does not affect motoneuron dendrite spacing but serves an essential, cell-autonomous function in new dendritic branch formation, thus adding a novel function of Dscam 1 in Drosophila CNS development.

\section{Materials and Methods}

Drosophila stocks. Drosophila melanogaster were reared in $68 \mathrm{ml}$ vials on a standard yeast corn meal medium at $25^{\circ} \mathrm{C}$, with a $12 \mathrm{~h}$ light/dark regiment and 60\% humidity (Duch et al., 2008). Male and female flies were used for experiments $1-2 \mathrm{~d}$ after eclosion. Gal4 driver lines are as follows: (1) ELAV(C155)-Gal4 was used for pan-neuronal expression and (2) C380-Gal4, UAS-mcd8-GFP;;Cha-GAL80 was used to restrict transgene expression to a subset of motoneurons and some other unidentified neurons (Budnik et al., 1996; Sanyal et al., 2003; Sanyal, 2009). The ChaGAL80 transgene inhibited expression in unidentified cholinergic sensory neurons and interneurons (Aberle et al., 2002), leaving expression in approximately 30 neurons per segment in the ventral nerve cord (for images of expression patterns see Boerner and Duch, 2010). To knock down Dscam1 under the control of C380-Gal4, UAS-mcd8-GFP;; Cha-GAL80, three different UAS-Dscam-RNAi transgenes were used [Vienna Drosophila RNAi Center (VDRC), fly stock numbers 36233, 108835, and 25623]. All three RNAi constructs were designed to target all Dscam 1 isoforms, and bioinformatics analysis as provided by the VDRC yielded no off-targets. In some experiments UAS-Dcr2 (Bloomington 24650) was coexpressed with UAS-Dscam-RNAi to enhance transgenic RNAi effects (Dietzl et al., 2007). Western blotting from whole brains with expression of either UAS-Dscam-RNAi or UAS-Dscam-RNAi and UAS-Dcr2 both under the control of the pan-neuronal driver Elav-GAL4 (C155) confirmed that inclusion of extra dicer enhanced knockdown strength by $\sim 20 \%$ from $\sim 30$ to $50 \%$ (data not shown). However, it remains unclear to which degree inclusion of extra dicer enhanced Dscam knockdown in MN5 when expressed under the control of the motoneuron driver C380-GAL4. Control data were derived from C380Gal4, UAS-mcd8-GFP;;Cha-GAL80 crossed to $\mathrm{w}^{1118}$ flies or UAS-Dcr2, both of which provide morphometric parameters in MN5 consistent with control strains that have been previously published (Vonhoff and
Duch, 2010; Vonhoff et al., 2012). Fly stocks for mutant motoneurons in an otherwise control background (MARCM) experiment were as follows: hsFLP, C155-Gal4, UAS-mCD8-GFP; FRT42D, tubP-GAL80/CyO (Matthews et al., 2007), FRT42D Dscam21/CyO (Hummel et al., 2003), and FRT42D Dscam47/CyO (Matthews et al., 2007; generous gifts from Wesley Gruber, Columbia University, New York).

Intracellular staining, image acquisition, and geometric reconstructions. Adult D. melanogaster were dissected and dye filled with sharp electrodes as described previously (Duch et al., 2008). For dendritic morphometric analysis, stacks of optical sections with $0.3 \mu \mathrm{m}$ thickness and $1024 \times 1024$ pixel resolution were acquired on a Leica TCS SP5 confocal laserscanning microscope with a $40 \times$ oil-immersion, 1.2 NA lens. Cy3streptavidin was excited at $568 \mathrm{~nm}$ (krypton laser) and emission was detected between 580 and $620 \mathrm{~nm}$. Cy2 was scanned with an excitation wavelength of $488 \mathrm{~nm}$ (argon laser) with emission detected between 495 and $530 \mathrm{~nm}$. Image stacks were further processed with AMIRA 4.1.1 software (TGS), and custom AMIRA plug-ins were used for geometric dendrite reconstructions as previously published (Schmitt al., 2004: Evers et al., 2005). For Sholl analysis the entire primary neurite was defined as tree origin, and the number of branches and total length were quantified within concentric spheres at $5 \mu \mathrm{m}$ intervals (Vonhoff and Duch, 2010).

MARCM analysis. We used mosaic analysis with a repressible marker (MARCM; Lee and Luo, 1999) to produce Dscam mutant MN5 in an otherwise control background. For MARCM clones, we crossed hsFLP, C155-Gal4, UAS-mCD8-GFP; FRT42D, tubP-GAL80/CyO to FRT42D Dscam $^{21} / \mathrm{CyO}$ or Dscam ${ }^{47} / \mathrm{CyO}$. Females were provided with a freshly yeasted grape agar plate and allowed to lay eggs for $2 \mathrm{~h}$. MN5 is born embryonically (Consoulas et al., 2002), but the exact time point is unknown. Therefore, embryos were collected and allowed to develop at $19^{\circ} \mathrm{C}$ before a heat shock $\left(37.5^{\circ} \mathrm{C}\right)$ was performed for $1 \mathrm{~h}$ at a variety of different time points during embryonic development. MN5 was visible in some preparations following heat shock after $9 \mathrm{~h}$ incubation $(n=5)$. The major limitation of this approach is a low yield.

We identified GFP-labeled clones by examining 1-d-old adult Drosophila under a fluorescence microscope. Selected preparations were immersed in cold saline, $\mathrm{pH}$ 7.4, opened along the dorsal ventral midline, fixed with $4 \%$ paraformaldehyde for $50 \mathrm{~min}$, and washed with PBS. Preparations were washed in PBS with $0.5 \%$ Triton X-100, PBS with $0.3 \%$ Triton X-100, and incubated overnight with rabbit anti-GFP (diluted 1:200; Invitrogen; A11122). After incubation with primary antibodies, the tissue was rinsed for several hours in PBS and incubated with Cy2conjugated goat anti-rabbit (diluted 1:500). The tissue was then rinsed in PBS, dehydrated in an ascending ethanol series (10 min each in 50, 70, 90, and $100 \%$ ), cleared, and mounted in methyl salicylate.

Statistical analysis. All statistical analyses were conducted with GraphPad Prism 6.0. Independent sample $t$ tests were used to analyze total dendritic length and dendritic branches. Parameters from the Sholl analysis were analyzed using a two-way ANOVA followed with Bonferroni post hoc tests, where applicable. All $p$ values $<0.05$ were considered significant. Graphical representations were prepared using CorelDraw13 (Corel) and GraphPad Prism 6.0.

\section{Results}

The mature MN5 is a large monopolar motoneuron that is located in mesothoracic neuromere of the adult Drosophila ventral nerve cord. It innervates the two dorsalmost fibers of the contralateral dorsal longitudinal flight muscle (DLM; Ikeda and Koenig, 1988; Fig. 1A, DLM fibers 5 and 6). Each of the four ventralmost DLM fibers is innervated by one motoneuron, namely MN1-4, with somata on the ipsilateral side of the ventral nerve cord. Its unique location makes MN5 unambiguously iden- 

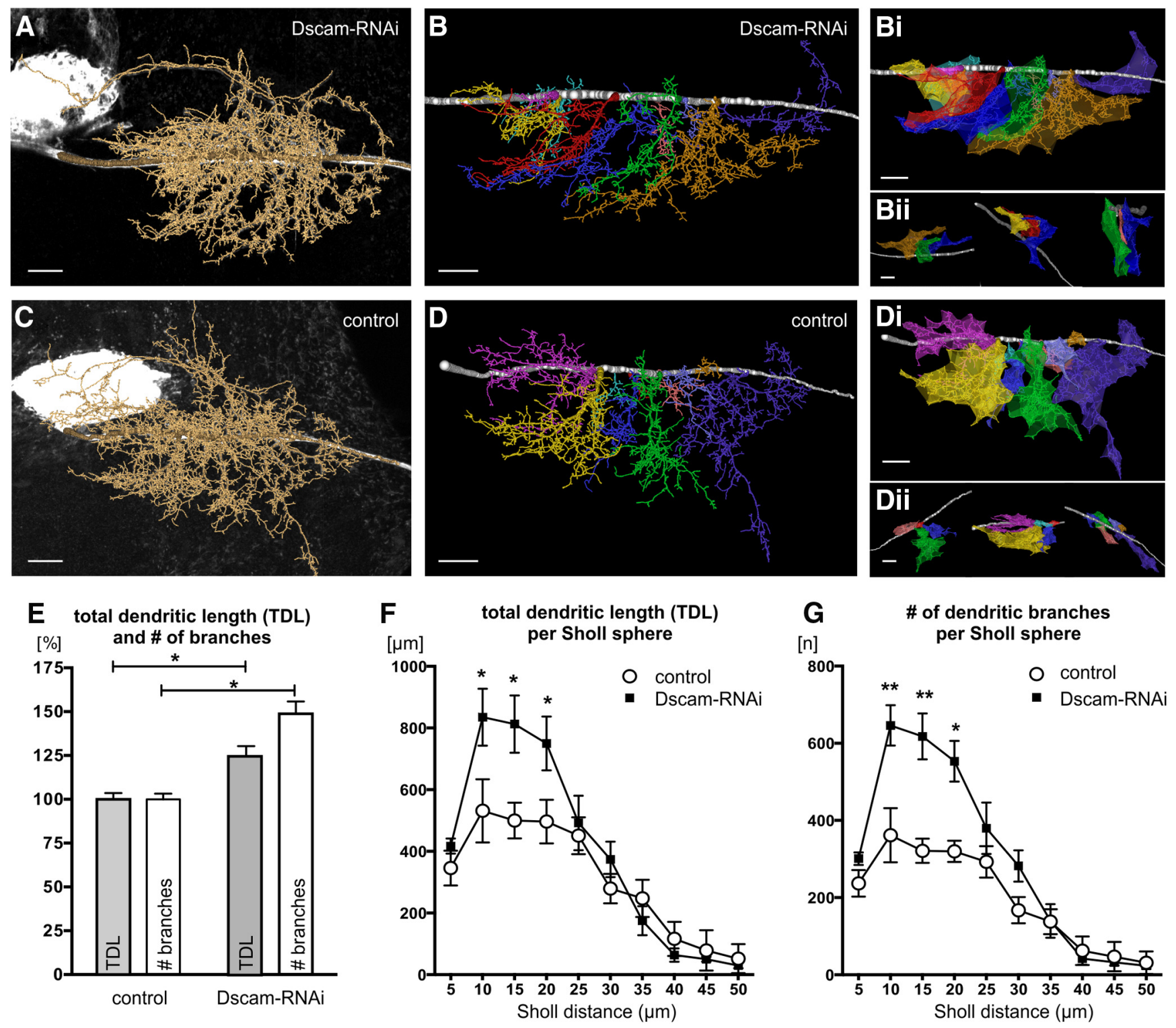

Figure 2. Expression of Dscam-RNAi in MN5 causes dendrite overgrowth. $A$, $C$, The fine structures of MN5 from (A) Dscam-RNAi (C380-GAL4, UAS-mcd8-GFP;;Cha-GAL80 $\times$ UAS-Dscam-RNAi) and $(\boldsymbol{C})$ control (C380-GAL4, UAS-mcd8-GFP;;Cha-GAL80 $\times$ W1118) are depicted as representative projection views superimposed with geometric reconstructions of MN5 dendrites. Ten dendritic subtrees that branch posteriorly from the primary neurite can be identified by the sequence of their origins and colored for visualization $(\boldsymbol{B}, \boldsymbol{D})$. To visualize individual subtrees, $3 \mathrm{D}$ colored volumes were wrapped around each subtree (Bi, Di) and visualized from different angles (Bii, $\mathbf{D i i})$. Individual dendritic subtrees were separated from each other in $3 \mathrm{D}$ space for both Dscam-RNAi (Bi, Bii) and control (Di, Dii). E, MN5 total dendritic length (TDL; gray bars) and number of branches (white bars) from control and Dscam-RNAi normalized to mean control values. $F$, $\mathbf{G}$, Sholl analysis of MN5 total dendritic length $(\boldsymbol{F})$ and number of branch points $(\boldsymbol{G})$ in control and following Dscam-RNAi knockdown. Each point represents mean \pm SEM; $n=3$ for control and Dscam-RNAi neurons $\left({ }^{*} p<0.05\right.$, ${ }^{* *} p<0.001$, two-way ANOVA with Bonferroni post test). Scale bars: $10 \mu \mathrm{m}$.

tifiable from animal to animal (Coggshall, 1978; Consoulas et al., 2002). MN5 has a complex dendritic tree (Fig. $1 B$ ) with $>6.5 \mathrm{~mm}$ of total length and approximately 4000 dendritic branches (Duch et al., 2008; Kuehn and Duch, 2013). MN5 sister dendrites are evenly spaced, show no self-contacts, and are distributed over 23 identifiable dendritic subtrees, which tile the input territory, i.e., dendritic branches of neighboring subtrees do not interdigitate, a phenomenon referred to as intraneuronal tiling (Vonhoff and Duch, 2010; Fig. 2).

Targeted Dscam-RNAi knockdown in MN5 does not affect dendrite spacing but causes overgrowth

To test for a potential role of Dscam 1 in motoneuron dendrite development we first sought to compare adult dendritic mor- phology of MN5 following Dscam-RNAi knockdown under the control of the motoneuron driver C380-GAL4, Cha-GAL80 (see Materials and Methods) with controls (C380-GAL4, Cha-GAL80 crossed to $\mathrm{w}^{1118}$ ). Dscam-RNAi knockdown in MN5 (Fig. 2A) had no obvious effects on its characteristic overall shape or the dendritic territory borders, as compared with controls (Fig. 2C). Furthermore, MN5 dendritic branches remained evenly spaced following Dscam knockdown and segregated into identifiable dendritic subtrees, which did not interdigitate. For visualization purposes the posterior dendritic subtrees are shown in different colors for a representative Dscam knockdown (Fig. 2B) and a representative control MN5 (Fig. 2D). Both Dscam-RNAi knockdown and controls displayed 10 posterior dendritic subtrees of variable sizes and projection areas, which confirmed previously 
published analyses (Vonhoff and Duch, 2010). Within each subtree the individual dendritic branches remained evenly spaced. Wrapping 3D volumes around each dendritic subtree and rotating these in space demonstrated that each dendritic subtree innervated a separate neuropil space and that different subtrees did not overlap or interdigitate for both Dscam-RNAi knockdown (Fig. 2Bi,Bii) and controls (Fig. 2Di,Dii). Therefore, DscamRNAi expression in MN5 had no effect on the overall dendritic shape, even spacing of dendrites, or intraneuronal tiling (Vonhoff and Duch, 2010) by different dendritic subtrees. These results were consistent for all three UAS-RNAi constructs tested (see Materials and Methods).

In contrast, quantification revealed significant increases of total dendritic length and branch numbers following Dscam-RNAi knockdown by $\sim 25$ and $40 \%$, respectively (Fig. $2 E$ ). Sholl analysis revealed that these increases were caused by additional dendritic branches close to the primary neurite (between 5 and 20 $\mu \mathrm{m}$ ), but more distal dendrites were not affected (Fig. $2 F, G$ ). In summary, expression of Dscam-RNAi in a subset of motoneurons did not affect dendritic spacing or intraneuronal tiling, but rather, caused significant increases in dendritic branching.

\section{Enhanced Dscam-RNAi knockdown does not affect dendrite spacing but impairs growth}

Typically, RNAi resembles a loss-of-function phenotype, but cannot be counted on to produce a null phenotype. Although RNAi transgenes have been effective in previous studies in the Drosophila nervous system (Yu et al., 2009), the specific knockdown efficacy in MN5 remained unknown. As such, we next increased the efficacy of the knockdown by coexpressing the UAS-Dscam-RNAi together with an extra copy of Dicer enzyme (UAS-Dcr2; Dietzl et al., 2007).

Overexpression of UAS-Dcr2 alone under the control of C380-GAL4 had no obvious effect on MN5 dendritic shape (Fig. $3 A$ ), dendrite spacing, or intraneuronal tiling, nor did it affect total dendritic length or the number of dendritic branches as compared with controls (Fig. 3D; $p>0.2$ ). In contrast, enhancing the efficacy of Dscam-RNAi knockdown by inclusion of UASDcr2 always caused a reduction in the number of dendritic branches. This dendritic defect occurred with different severities. In $40 \%$ of the preparations examined ( $n=10$ of 25 ), we observed the normal number of 23 dendritic subtrees within the adult MN5, which tiled the input space and did not interdigitate. Branches within each subtree were spaced evenly. However, total dendritic length and the number of branches were significantly reduced to about half of control values (Fig. $3 B, D$ ). Sholl analysis demonstrated significant reductions in the number of branches and total dendritic length in all distances between 10 and $30 \mu \mathrm{m}$ from the primary neurite (Fig. $3 E, F$ ), a Sholl region that contains $\sim 70 \%$ of all MN5 dendrites (Vonhoff and Duch, 2010). The other $60 \%$ of MN5 preparations with coexpression of DscamRNAi and Dcr2 ( $n=15$ of 25$)$ displayed a complete lack of all mature dendrites (Fig. 3C). Many densely packed lamellipodialike and filopodia-like structures branched off the primary neurite, but few processes extended further than $20 \mu \mathrm{m}$ from the primary neurite. This phenotype was highly stereotyped between all 15 preparations. Although no off-targets are predicted for the UAS-RNAi construct used (VDRC 36233), we repeated these experiments with two additional UAS-RNAi constructs (VDRC 108835 and 25623) to account for possible nonspecific RNAi effects. No matter which of the three UAS-RNAi constructs was used, coexpression of Dscam-RNAi and Dcr2 yielded either a reduction in the number of dendrites or a complete lack of all adult dendrites and lamellipodia-like structures branching off the primary neurite (Fig. 3, compare $C, G, H$ ).

In principle, a lack of adult dendrites following Dscam1 RNAi knockdown could either be caused by a loss of previously grown dendrites, of by deficient new dendrite formation. To test whether dendrites ever formed during development, we also dye filled MN5 at early stages of dendrite growth. Dendrite growth begins at pupal stage P5 (Vonhoff et al., 2013). In controls, primary and many secondary dendritic branches have already formed at pupal stage P6, and many thin filopodia-like structures extend from these newly formed dendrites (Fig. 3I). By pupal stage $\mathrm{P} 9$, many high-order dendrites have formed (Fig. $3 K$ ). In contrast, following Dscam-RNAi and Dcr2 expression $\sim 60 \%$ of all pupal MN5s displayed only lamellipodia-like and filopodialike structures at either pupal stage P6 (Fig. 3J), or at pupal stage P9 (Fig. $3 L$ ), similar to our results in the adult. This showed that the lack of adult dendrites was caused by a deficiency in dendrite formation and not by a loss of previously formed dendrites.

These data suggested a role for Dscam 1 in motoneuron dendritic branch formation during normal development, which was not in accord with the current understanding that Dscam operates as a cell adhesion molecule that mediates homophilic repulsion. However, RNAi knockdown usually does not yield a complete loss of function phenotype. Accordingly, some Dscam protein is left in MN5 following knockdown, and the amount needed to mediate normal repulsion remains unknown. In addition, despite inclusion of Cha-GAL80 to suppress expression in cholinergic neurons, C380 also expresses in approximately 30 unidentified neurons in the mesothoracic neuromere of the ventral nerve cord (Boerner and Duch, 2010). Therefore, our RNAi experiments did not exclude the possibility of indirect effects via loss of homophilic repulsion in other yet unidentified neurons. As such, we conducted two sets of experiments to address this question. First, we repeated RNAi knockdown experiments with an additional GAL4 driver (D42; Sanyal, 2009) that expresses in many motoneurons and a set of approximately 40 additional neurons in the mesothoracic neuromere, which are mostly nonoverlapping as compared with C380-GAL4 expression patterns (Boerner and Duch, 2010). These experiments yielded results comparable (data not shown) to those reported above for C380GAL4, indicating that Dscam may exert direct action on normal motoneuron dendritic growth. Second, we used the MARCM technique to produce Dscam mutant MN5s in an otherwise normal Dscam background.

\section{Dscam mutant motoneurons have no dendrites}

To test whether Dscam was required in a cell-autonomous manner for normal motoneuron postembryonic dendritic growth, we produced mutant MN5s in a control background. We used a heat shock flipase to induce homologous recombination at the time of MN5 birth. Since the precursor cell and the exact time point of MN5 birth are unknown, this strategy had a low yield with approximately 1 of 100 animals carrying a mutant MN5 in a control-like background. However, we identified four animals with a Dscam ${ }^{47}$ mutant MN5. Dscam ${ }^{47}$ carries a frame shift in the cytoplasmic domain, which in turn, causes modest loss of function (Matthews et al., 2007). All four MN5s displayed no dendrites, but rather, small stump-like processes branching of the primary neurite (Fig. $4 B$ ). In addition we tested Dscam ${ }^{21}$, a strong loss of function allele for which no protein was detected in Western blotting (Hummel et al., 2003). Five Dscam ${ }^{21}$ mutant MN5s also had no dendrites, but both the somata and the primary neurites were surrounded by densely packed lamellipodia- 

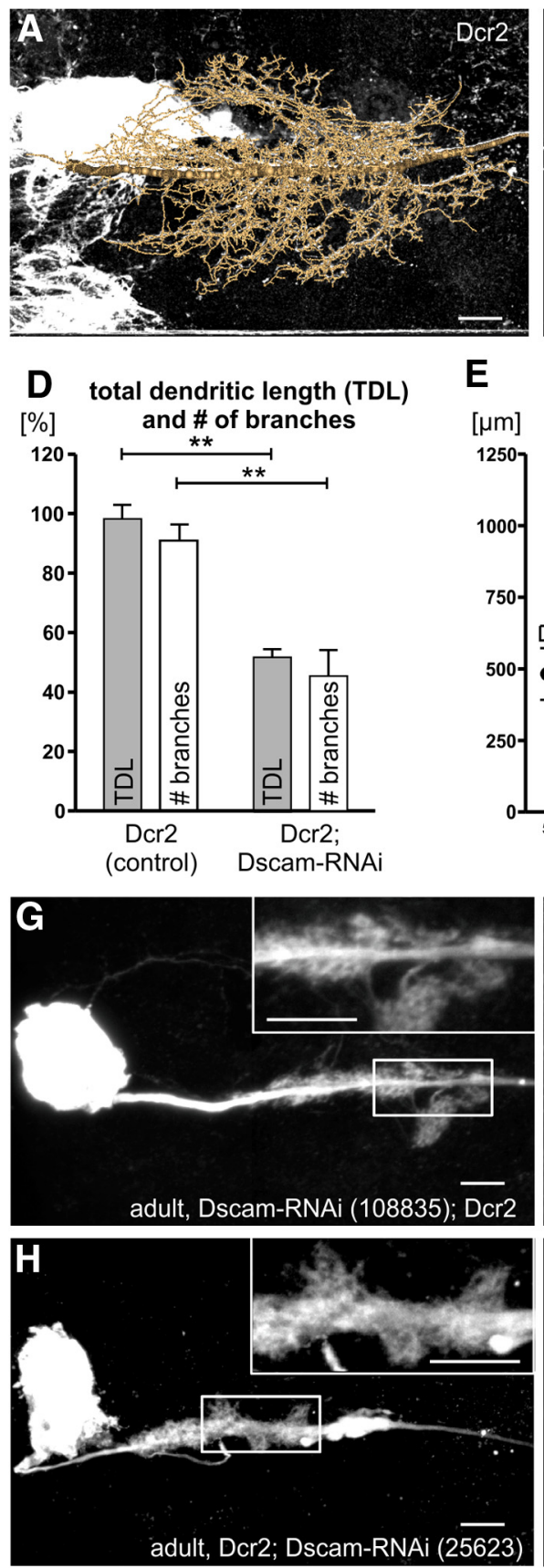

E
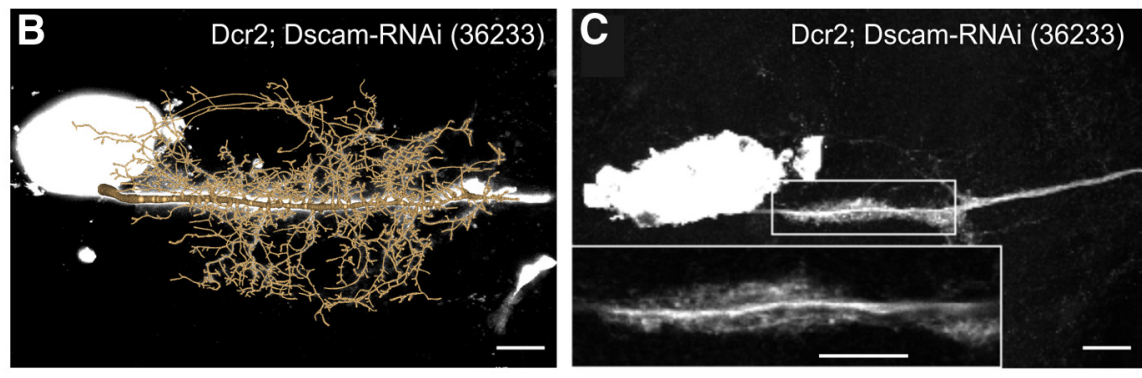

total dendritic length (TDL) per Sholl sphere

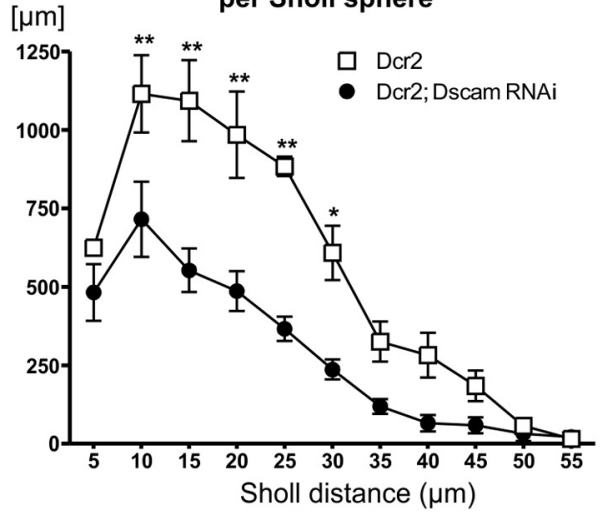

$\mathbf{F}$

[n]

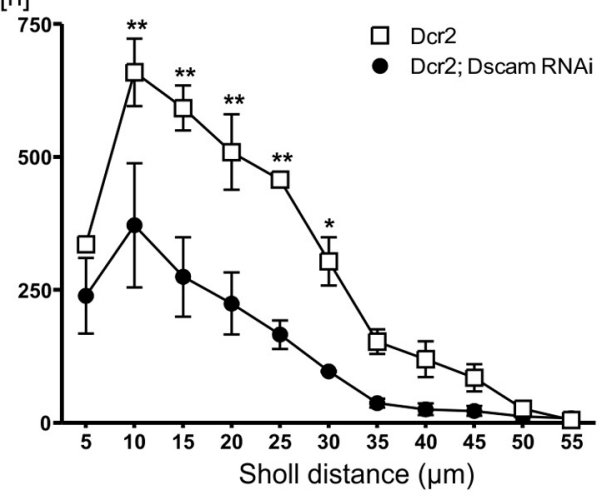

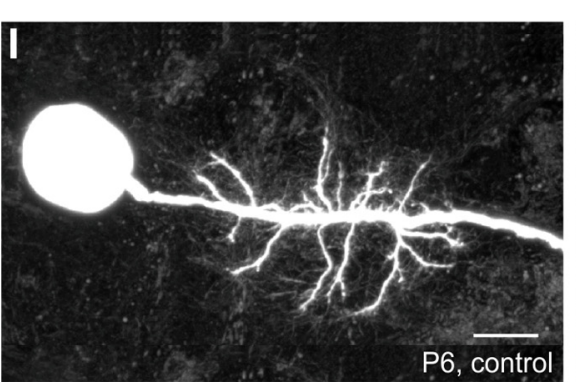
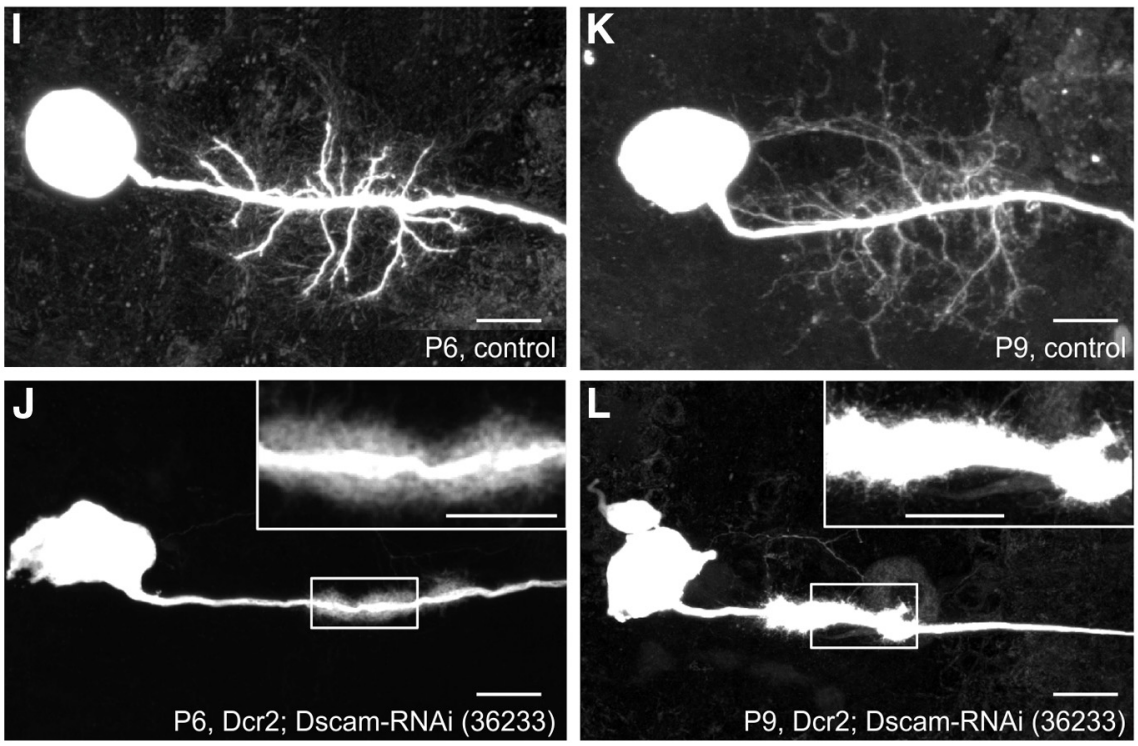

Figure 3. Enhanced Dscam-RNAi knockdown in MN5 impairs dendrite growth. A-C, Representative dendrite reconstruction of MN5 following expression of UAS-Dcr2 alone (C380-GAL4, UAS-mcd8-GFP;; Cha-GAL80 × UAS-Dcr2; $A$ ), and following targeted expression of Dscam-RNAi and Dcr2 in MN5 (C380-GAL4, UAS-mcd8-GFP;;'Cha-GAL80 X UAS-Dcr2; UAS-Dscam-RNAi (VDRC lines \#36233; $\boldsymbol{B}, \boldsymbol{C})$, which caused a reduction in dendritic branches in 10 of 25 preparations $(\boldsymbol{B})$ and a complete lack of dendrites in 15 of 25 preparations (C). D, Total dendritic length (TDL; gray bars) and number of branches (white bars) in MN5 following expression of Dcr2 alone compared with expression of Dscam-RNAi and Dcr2, both normalized to mean control values. $\boldsymbol{E}, \boldsymbol{F}$, Sholl analysis of TDL $(\boldsymbol{E})$ and number of branches $(\boldsymbol{F})$. MN5 preparations without dendrites $(\boldsymbol{C}, \boldsymbol{G})$ were not included in quantification shown in $\boldsymbol{D}-\boldsymbol{F}$. Each point represents mean $\pm S E M$. Dcr2, $n=3$ neurons, Dcr2; Dscam-RNAi, $n=3$ neurons ( ${ }^{*} p<0.05$, ${ }^{* *} p<0.001$ two-way ANOVA with Bonferroni post test). $\boldsymbol{G}, \boldsymbol{H}$. Representative projection views of adult MN5 dendritic structure following expression of two different Dscam-RNAi constructs, each together with and Dcr2. I-L, Representative projection views of MN5 at pupal stages P6 and P9 in controls $(\boldsymbol{I}, \boldsymbol{K})$ and following expression of Dscam-RNAi and Dcr2 $(J, L)$. White boxes depict selective enlargements of filopodia-like and lamellipodia-like processes. Scale bars: $10 \mu \mathrm{m}$.

like and filopodia-like processes (Fig. 4C). This corresponded with the lack of dendrites in MN5 following targeted expression of Dscam-RNAi and Dcr2 (Fig. 3C,G-I) suggesting that Dscam1 may be required for MN5 dendrite growth in a cell-autonomous manner during normal development.

While screening through hundreds of nervous systems in MARCM flies we often observed additional unidentified neurons with qualitatively similar dendritic phenotypes (Fig. $4 D-F$ ). Other unidentified motoneurons with axons projecting into thoracic nerves showed filopodia-like and lamellipodia-like processes instead of dendritic ramifications (Fig. 4D,E). Occasionally, we also identified some neurons with similarly clumped processes as interneurons because they had somata in the thoracic CNS but no efferent axons (Fig. $4 F$ ). This indicated that Dscaml may be required for normal dendrite growth in multiple types of motoneurons and in some types of interneurons. 

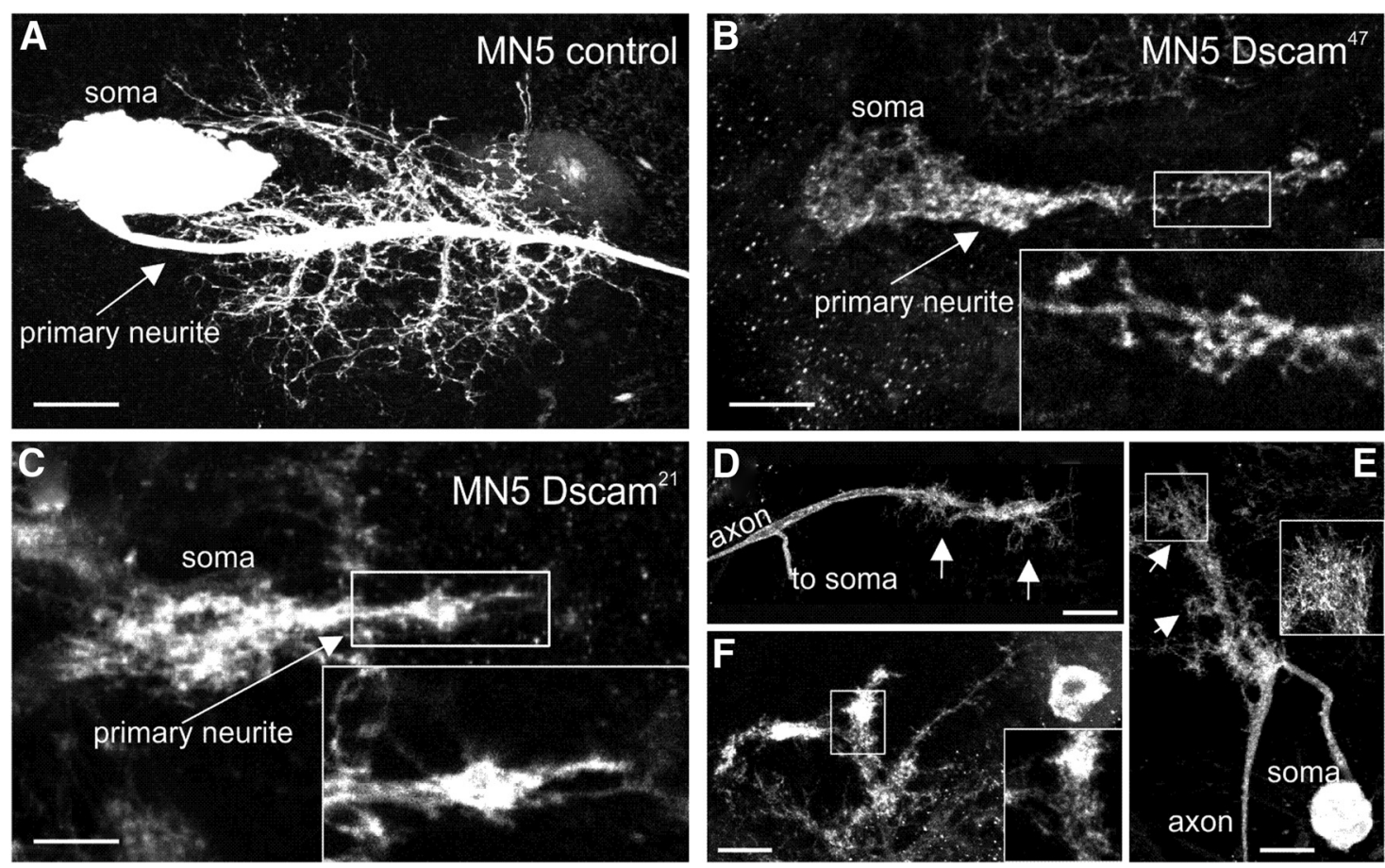

Figure 4. Dscam is required cell autonomously for dendrite growth in MN5. $\boldsymbol{A}-\boldsymbol{C}$, Representative projection views of a control MN5 $(\boldsymbol{A})$ as compared with Dscam ${ }^{47}(\boldsymbol{B})$ and Dscam ${ }^{21}(\boldsymbol{C})$ mutant MN5s. Boxes indicate selectively enlarged areas with lamellipodia-like and filopodia-like processes. $\mathbf{D}-\boldsymbol{F}$, Unidentified Dscam ${ }^{47}$ mutant neurons. Dendritic regions of unidentified thoracic motoneurons show filopodia- and lamellipodia-like structures (see white arrows in $\mathbf{D}, \boldsymbol{E}$ ). Similarly, central arbors of a nonidentified thoracic interneuron show clumped lamellipodia-like structures (F). Scale bars: $20 \mu \mathrm{m}$.

\section{Discussion}

Our key finding is that Dscam1 may not be required for selfrecognition, self-repulsion, and even spacing of adult motoneuron dendrites, but rather, may be essential for dendritic growth and branching. This imparts a novel function to Drosophila Dscam 1, which is commonly viewed as an NCAM that operates via homophilic repulsion to mediate self-avoidance (Matthews et al., 2007; Soba et al., 2007), dendritic patterning (Zhu et al., 2006), and postsynaptic specificity (Millard et al., 2010). However, recent findings from mouse pyramidal neurons indicate functions of vertebrate Dscam in dendritic arbor growth and spine formation (Maynard and Stein, 2012). Our data indicate a conserved role of Dscam in central neuron dendritic arbor growth and branching.

\section{Dscam 1 loss of function does not impair spacing but affects dendritic growth}

Targeted expression of Dscam-RNAi in MN5 resulted in significant increases in dendritic branch number and total dendritic length without disrupting dendritic spacing or intraneuronal tiling. No dendritic crossings were observed, and the characteristic organization of MN5 into 23 identifiable dendritic subtrees, which tile the neuropil space and avoid interdigitation (Vonhoff and Duch, 2010), was not affected. These data indicated that Dscam might serve an alternative function in motoneuron dendrites (this study) compared with mechanosensory neuron dendrites, where Dscam functions to space dendrites via homophilic repulsion (Hughes et al., 2007; Matthews et al., 2007; Soba et al., 2007). One major difference between sensory neuron dendrites and motoneuron dendrites is that the latter receive synaptic input.

Recent findings demonstrate that Dscam protein levels serve as instructive codes for regulating axon targeting and arbor size, and further, that Dscam translation is tightly regulated by leucine zipper kinase and FMRP (Cvetkovska et al., 2013; Kim et al., 2013). Together with the inability of RNAi to produce a true null phenotype, these observations suggest that it would be difficult to isolate the observed dendritic overgrowth to reductions in Dscam protein in MN5. To address these limitations, we explored an alternative strategy to test for potential roles of Dscam in motoneuron dendritic growth. First, we enhanced the efficacy of the RNAi knockdown by coexpression of an extra Dicer enzyme (Dcr2; Dietzl et al., 2007). We considered this to be a promising technique because pan-neuronal expression of UAS-DscamRNAi with UAS-Dcr2 under the control of Elav-GAL4 (C155GAL4; Lin and Goodman, 1994) caused significant reductions in vitality (data not shown), similar to strong loss of function alleles in Dscam mutant flies (Hummel et al., 2003; Hattori et al., 2007), whereas pan-neuronal expression of UAS-Dcr2 alone has no obvious effect on vitality. Second, we generated Dscam single mutant MN5 neurons in a control background. Following enhanced Dscam-RNAi knockdown, $60 \%$ of all adult MN5s were devoid of all mature dendrites. Similarly, all Dscam ${ }^{47}$ and Dscam ${ }^{21}$ mutant MN5s were devoid of mature dendritic branches. In some animals, dendrites were formed following targeted expression of Dscam-RNAi with Dcr2, but these possessed significantly fewer dendrites than controls. We conclude that significant loss of Dscam function (60\% of all animals with Dcr2 and Dscam-RNAi, Dscam $^{47}$, and Dscam ${ }^{21}$ ) prevented stable dendrites from being formed. Instead, dense filopodia-like and lamellipodia-like processes were observed along the primary neurite that represents the root of 23 elaborate dendritic subtrees in control MN5 (Vonhoff and Duch, 2010). During normal development filopodialike and lamellipodia-like structures are formed during early stages of dendritic growth (pupal stages P5 and P6; Vonhoff et al., 2013). In the absence of Dscam 1 , a dense meshwork of filopodia 
and lamellipodia is formed at these stages, but these structures do not develop into mature dendrites at any stage of development. This indicates that Dscam1 may be required for the transformation of actin-rich filopodia into stable dendrites, though the underlying mechanism remains unknown.

\section{Potential mechanism of Dscam action during early dendritic growth}

The mechanisms by which Dscam functions either in selfavoidance or in dendrite formation remain unknown on the cellular and the molecular level. In Drosophila, the tremendous molecular diversity of Dscam 1 provides each neuron with its own cell surface identity (Neves et al., 2004). Biochemical evidence demonstrates homophilic binding among isoforms that share the same extracellular domain, but little to no interaction between other isoforms (Wojtowicz et al., 2004). In neurons, interactions between identical Dscam isoforms promote contactdependent repulsion (Wang et al., 2002; Wojtowicz et al., 2004). Contact-dependent repulsion among self-dendrites has been suggested to affect dendritic arbor size, and it is conceivable that self-avoidance among sister dendrites will promote elaboration though the receptive field (Zhu et al., 2006). Accordingly, loss of Dscam function causes smaller dendritic fields of Drosophila OPNs, whereas overexpression of Dscam results in more diffuse and widespread dendrites (Zhu et al., 2006). Loss of Dscam function also caused reduced dendritic arbor growth in the early developing cerebral cortex (Maynard and Stein, 2012). However, a lack of self-repulsion seems unlikely to underlie the filopodiaand lamellipodia-like phenotype observed for motoneuron dendritic growth in the present study, because dendritic surface as a substrate for contact repulsion is never formed. We can, of course, not exclude the possibility that actin-rich filopodia and lamellipodia during early stages of dendritic growth may exhibit cell-type intrinsic adhesive cues, which are masked by the presence of Dscam protein during normal development, so that they are prevented from outgrowth in the absence of Dscam protein. However, this hypothesis is not consistent with our observation that a mild reduction of Dscam in MN5 (RNAi without extra Dicer) caused dendritic overgrowth. Therefore, we favor the idea that Dscam affects intracellular signaling pathways that regulate dendritic growth cone dynamics, such as small Rho GTPase signaling (Van Aelst and Cline, 2004). The intracellular domains of fly and human Dscams interact with P21 activated kinase (PAK1; Schmucker et al., 2000; Li and Guan, 2004), which in turn, provides a possible mechanistic link to Rho GTPases and actin polymerization. Since PAK1 is not required for self-avoidance (Andrews et al., 2008), it may be a candidate for Dscam-mediated dendritic branch formation as described in this study, but this is currently speculation.

In summary, in both vertebrates and invertebrates Dscam serves a variety of different neurodevelopmental functions depending upon the neuron type and species under investigation. Together with recent data on Dscam function in developing pyramidal neuron dendrites in murine hippocampus and cortex (Alves-Sampaio et al., 2010; Maynard and Stein, 2012), this study suggests a conserved function of Dscam in new dendritic branch formation during early stages of neuronal differentiation.

\section{References}

Aberle H, Haghighi AP, Fetter RD, McCabe BD, Magalhães TR, Goodman CS (2002) Wishful thinking encodes a BMP type II receptor that regulates synaptic growth in Drosophila. Neuron 33:545-558. CrossRef Medline

Alves-Sampaio A, Troca-Marín JA, Montesinos ML (2010) NMDA-mediated regulation of DSCAM dendritic local translation is lost in a mouse model of Down's syndrome. J Neurosci 30:13537-13548. CrossRef Medline

Andrews GL, Tanglao S, Farmer WT, Morin S, Brotman S, Berberoglu MA, Price H, Fernandez GC, Mastick GS, Charron F, Kidd T (2008) Dscam guides embryonic axons by Netrin-dependent and -independent functions. Development 135:3839-3848. CrossRef Medline

Boerner J, Duch C (2010) Average shape standard atlas for the adult Drosophila ventral nerve cord. J Comp Neurol 518:2437-2455. CrossRef Medline

Budnik V, Koh YH, Guan B, Hartmann B, Hough C, Woods D, Gorczyca M (1996) Regulation of synapse structure and function by the Drosophila tumor suppressor gene dlg. Neuron 17:627-640. CrossRef Medline

Cline HT (2001) Dendritic arbor development and synaptogenesis. Curr Opin Neurobiol 11:118-126. CrossRef Medline

Coggshall JC (1978) Neurons associated with the dorsal longitudinal flight muscles of D. melanogaster. J Comp Neurol 177:707-720. Medline

Consoulas C, Restifo LL, Levine RB (2002) Dendritic remodeling and growth of motoneurons during metamorphosis of Drosophila melanogaster. J Neurosci 22:4906-4917. Medline

Cooke BM, Woolley CS (2005) Gonadal hormone modulation of dendrites in the mammalian CNS. J Neurobiol 64:34-46. CrossRef Medline

Cvetkovska V, Hibbert AD, Emran F, Chen BE (2013) Overexpression of Down syndrome cell adhesion molecule impairs precise synaptic targeting. Nat Neurosci 16:677-682. CrossRef Medline

Dietzl G, Chen D, Schnorrer F, Su KC, Barinova Y, Fellner M, Gasser B, Kinsey K, Oppel S, Scheiblauer S, Couto A, Marra V, Keleman K, Dickson BJ (2007) A genome-wide transgenic RNAi library for conditional gene inactivation in Drosophila. Nature 448:151-156. CrossRef Medline

Duch C, Mentel T (2004) Activity affects dendritic shape and synapse elimination during steroid controlled dendritic retraction in Manduca sexta. J Neurosci 24:9826-9837. CrossRef Medline

Duch C, Vonhoff F, Ryglewski S (2008) Dendrite elongation and dendritic branching are affected separately by different forms of intrinsic motoneuron excitability. J Neurophysiol 100:2525-2536. CrossRef Medline

Evers JF, Schmitt S, Sibila M, Duch C (2005) Progress in functional neuroanatomy: precise automatic geometric reconstruction of neuronal morphology from confocal image stacks. J Neurophysiol 93:2331-2342. CrossRef Medline

Fuerst PG, Koizumi A, Masland RH, Burgess RW (2008) Neurite arborization and mosaic spacing in the mouse retina require DSCAM. Nature 451:470-474. CrossRef Medline

Fuerst PG, Bruce F, Tian M, Wei W, Elstrott J, Feller MB, Erskine L, Singer JH, Burgess RW (2009) DSCAM and DSCAML1 function in self-avoidance in multiple cell types in the developing mouse retina. Neuron 64:484497. CrossRef Medline

Grueber WB, Jan LY, Jan YN (2002) Tiling of the Drosophila epidermis by multidendritic sensory neurons. Development 129:2867-2878. Medline

Hattori D, Demir E, Kim HW, Viragh E, Zipursky SL, Dickson BJ (2007) Dscam diversity is essential for neuronal wiring and self-recognition. Nature 449:223-227. CrossRef Medline

Hughes ME, Bortnick R, Tsubouchi A, Bäumer P, Kondo M, Uemura T, Schmucker D (2007) Homophilic Dscam interactions control complex dendrite morphogenesis. Neuron 54:417-427. CrossRef Medline

Hummel T, Vasconcelos ML, Clemens JC, Fishilevich Y, Vosshall LB, Zipursky SL (2003) Axonal targeting of olfactory receptor neurons in Drosophila is controlled by Dscam. Neuron 37:221-231. CrossRef Medline

Ikeda K, Koenig JH (1988) Morphological identification of the motor neurons innervating the dorsal longitudinal flight-muscle of Drosophila melanogaster. J Comp Neurol 273:436-444. Medline

Kim JH, Wang X, Coolon R, Ye B (2013) Dscam expression levels determine presynaptic arbor sizes in Drosophila sensory neurons. Neuron 78:827838. CrossRef Medline

Kim S, Chiba A (2004) Dendritic guidance. Trends Neurosci 27:194-202. CrossRef Medline

Koch C, Segev I (2000) The role of single neurons in information processing. Nat Neurosci [3 Suppl]:1171-1177. CrossRef Medline

Kramer AP, Kuwada JY (1983) Formation of the receptive fields of leech mechanosensory neurons during embryonic development. J Neurosci 3:2474-2486. Medline

Kramer AP, Stent GS (1985) Developmental arborization of sensory neurons in the leech Haementeria ghilianii. II. Experimentally induced variations in the branching pattern. J Neurosci 5:768-775. Medline 
Kuehn C, Duch C (2012) Putative excitatory and putative inhibitory inputs are localised in different dendritic domains in a Drosophila flight motoneuron. Eur J Neurosci 37:860-875. CrossRef Medline

Lee T, Luo L (1999) Mosaic analysis with a repressible cell marker for studies of gene function in neuronal morphogenesis. Neuron 22:451-461. CrossRef Medline

Li W, Guan KL (2004) The Down syndrome cell adhesion molecule (DSCAM) interacts with and activates Pak. J Biol Chem 279:32824-32831. CrossRef Medline

Libersat F, Duch C (2004) Mechanisms of dendritic maturation. Mol Neurobiol 29:303-320. Medline

Lin DM, Goodman CS (1994) Ectopic and increased expression of Fasciclin II alters motoneuron growth cone guidance. Neuron 13:507-523. CrossRef Medline

Liu G, Li W, Wang L, Kar A, Guan KL, Rao Y, Wu JY (2009) DSCAM functions as a netrin receptor in commissural axon pathfinding. Proc Natl Acad Sci U S A 106:2951-2956. CrossRef Medline

London M, Häusser M (2005) Dendritic computation. Annu Rev Neurosci 28:503-532. CrossRef Medline

Ly A, Nikolaev A, Suresh G, Zheng Y, Tessier-Lavigne M, Stein E (2008) DSCAM is a netrin receptor that collaborates with DCC in mediating turning responses to netrin-1. Cell 133:1241-1254. CrossRef Medline

Matthews BJ, Kim ME, Flanagan JJ, Hattori D, Clemens JC, Zipursky SL, Grueber WB (2007) Dendrite self-avoidance is controlled by Dscam. Cell 129:593-604. CrossRef Medline

Maynard KR, Stein E (2012) DSCAM contributes to dendrite arborization and spine formation in the developing cerebral cortex. J Neurosci 32: 16637-16650. CrossRef Medline

Millard SS, Lu Z, Zipursky SL, Meinertzhagen IA (2010) Drosophila dscam proteins regulate postsynaptic specificity at multiple-contact synapses. Neuron 67:761-768. CrossRef Medline

Montague PR, Friedlander MJ (1989) Expression of an intrinsic growth strategy by mammalian retinal neurons. Proc Natl Acad Sci U S A 86: 7223-7227. CrossRef Medline

Neves G, Zucker J, Daly M, Chess A (2004) Stochastic yet biased expression of multiple Dscam splice variants by individual cells. Nat Genet 36:240-246. CrossRef Medline

Polleux F, Morrow T, Ghosh A (2000) Semaphorin 3A is a chemoattractant for cortical apical dendrites. Nature 404:567-573. CrossRef Medline

Sanyal S (2009) Genomic mapping and expression patterns of C380, OK6 and D42 enhancer trap lines in the larval nervous system of Drosophila. Gene Expr Patterns 9:371-380. CrossRef Medline

Sanyal S, Narayanan R, Consoulas C, Ramaswami M (2003) Evidence for cell autonomous AP1 function in regulation of Drosophila motor-neuron plasticity. BMC Neurosci 4:20. CrossRef Medline

Schmitt S, Evers JF, Duch C, Scholz M, Obermayer K (2004) New methods for the computer-assisted 3-D reconstruction of neurons from confocal image stacks. Neuroimage 23:1283-1298. CrossRef Medline

Schmucker D, Clemens JC, Shu H, Worby CA, Xiao J, Muda M, Dixon JE, Zipursky SL (2000) Drosophila Dscam is an axon guidance receptor exhibiting extraordinary molecular diversity. Cell 101:671-684. CrossRef Medline
Scott EK, Reuter JE, Luo L (2003) Dendritic development of Drosophila high order visual system neurons is independent of sensory experience. BMC Neurosci 4:14. CrossRef Medline

Soba P, Zhu S, Emoto K, Younger S, Yang SJ, Yu HH, Lee T, Jan LY, Jan YN (2007) Drosophila sensory neurons require Dscam for dendritic selfavoidance and proper dendritic field organization. Neuron 54:403-416. CrossRef Medline

Spatkowski G, Schilling K (2003) Postnatal dendritic morphogenesis of cerebral basket and stellate cells in vitro. J Neurosci Res 72:317-326. CrossRef Medline

Sweeney NT, Li W, Gao FB (2002) Genetic manipulation of single neurons in vivo reveals specific roles of Flamingo in neuronal morphogenesis. Dev Biol 247:76-88. CrossRef Medline

Toran-Allerand CD, Singh M, Sétáló G Jr (1999) Novel mechanisms of estrogen action in the brain: new players in an old story. Front Neuroendocrinol 20:97-121. CrossRef Medline

Van Aelst L, Cline HT (2004) Rho GTPases and activity-dependent dendrite development. Curr Opin Neurobiol 14:297-304. CrossRef Medline

Vonhoff F, Duch C (2010) Tiling among stereotyped dendritic branches in an identified Drosophila motoneuron. J Comp Neurol 518:2169-2185. CrossRef Medline

Vonhoff F, Williams A, Ryglewski S, Duch C (2012) Drosophila as a model for MECP2 gain of function in neurons. PLoS One 7:e31835. CrossRef Medline

Vonhoff F, Kuehn C, Blumenstock S, Sanyal S, Duch C (2013) Temporal coherency between receptor expression, neural activity and AP-1dependent transcription regulates Drosophila motoneuron dendrite development. Development 140:606-616. CrossRef Medline

Wang J, Zugates C, Liang IH, Lee T (2002) Drosophila Dscam is required for divergent segregation of sister branches and suppresses ectopic bifurcation of axons. Neuron 33:559-571. Medline

Weeks JC, Levine RB (1995) Steroid hormone effects on neurons subserving behavior. Curr Opin Neurobiol 5:809-815. CrossRef Medline

Williams ME, de Wit J, Ghosh A (2010) Molecular mechanisms of synaptic specificity in developing neural circuits. Neuron 68:9-18. CrossRef Medline

Wojtowicz WM, Wu W, Andre I, Qian B, Baker D, Zipursky SL (2007) A vast repertoire of Dscam binding specificities arises from modular interactions of variable Ig domains. Cell 130:1134-1145. CrossRef Medline

Wong RO, Ghosh A (2002) Activity-dependent regulation of dendritic growth and patterning. Nat Rev Neurosci 3:803-812. CrossRef Medline

Yamagata M, Sanes JR (2008) DSCAM and Sidekick proteins direct laminaspecific synaptic connections in vertebrate retina. Nature 451:465-469. CrossRef Medline

Yu HH, Yang JS, Wang J, Huang Y, Lee T (2009) Endodomain diversity in the Drosophila Dscam and its roles in neuronal morphogenesis. J Neurosci 29:1904-1914. CrossRef Medline

Zhu H, Hummel T, Clemens JC, Berdnik D, Zipursky SL, Luo L (2006) Dendritic patterning by Dscam and synaptic partner matching in the Drosophila antennal lobe. Nat Neurosci 9:349-355. CrossRef Medline 